\title{
CHECKLIST FOR COMMERCIALLY IMPORTANT FOOD FISHES OF PARANGIPETTAI, SOUTHEAST COAST OF INDIA
}

\author{
Kudus Kadharsha1 ${ }^{*}$, Syed Ajmal Khan'1, Somasundharanair Lyla1, Ponnuvel Mohanchander ${ }^{1}$, Akbar John² \\ ${ }^{1}$ Annamalai University, Faculty of Marine Sciences, Centre of Advanced Study in Marine Biology, Parangipettai-608502, Tamil Nadu, India. \\ ${ }^{2}$ INOCEM, Kulliyyah of Science, International Islamic University Malaysia, Kuantan Pahang Malaysia. \\ *Corresponding author E-mail: kadharsha87@gmail.com
}

\section{ARTICLE DETAILS}

\section{Article History:}

Received 12 November 2017 Accepted 12 December 2017 Available online 1 January 2018

Keywords:

food fishes, taxonomical, Southeast coast

\section{ABSTRACT}

A checklist for commercially important food fishes from Parangipettai, Southeast coast of India is presented (lat. 11 $30^{\prime} \mathrm{N}$, long. $79^{\prime} 46^{\prime} \mathrm{E}$ ). This comprises 93 species belonging to 57 genera and 42 families currently known from the area, which are all of commercially valuable. We provided a synthesis of the taxonomical work carried out until now that could guide to future research..

\section{INTRODUCTION}

Fish forms a vital source of food and is man's important source of high quality protein, providing $16 \%$ of the animal protein consumed by the world's population (FA0, 2000) [1]. Marine fisheries have emerged as one of the largest industries in the country employing about 10 million people in 3,651 fishing villages along the $8,129 \mathrm{Km}$ coastline of India earning foreign exchange of over 7250 crores in 2009-2010.

India has an EEZ of 2.02 million $\mathrm{Km}^{2}$ and is endowed with a rich variety of demersal fishery resources. The exploited demersal finfish resources increased from $0.75 \mathrm{mt}$ in 1985 to register the peak of $1.35 \mathrm{mt}$ in 1998 . However, it decreased to $1.15 \mathrm{mt}$ in 2004 (Srinath et al., 2006) [2]. About 700 species of finfish have been recorded from the Indian sea bottom of which about 250 are common to the demersal fisheries. Species richness of demersal is more off the east coast than off the west coast (Bensam, 2000) [3].

Tamil Nadu has a coastline of about $1,076 \mathrm{Km}(13 \%$ of the country's coastline), 1.9 lakh $\mathrm{Km}^{2}$ of Exclusive Economic Zone (9\% of India's EEZ) and a continental shelf of about $41,412 \mathrm{Km}^{2}$. Tamil Nadu alone produced 363,000 tons of marine fish and 112,00 tons of inland fish amounting altogether to 475,000 tons of total fish in the year 2008 (total for the whole India 2.8 million tons).

In the southeast coast of India, Parangipettai waters where the present swot was carried out harbour a variety of food fishes, shrimps and other shellfishes. The fishes landed daily were marketed at the local fish market and in the surrounding villages and the choice items were moved to interior places so also exported. The surplus is sundried. As a wide range of fin fishes were contributing in the catch, hence a checklist has been made for the commercially important food fishes occurring in the waters of Parangipettai (southeast coast of India).

\section{MATERIALS AND METHODS}

\subsection{Study site}

Parangipettai (lat. 11 30' N, long. 79 46' E) situated on the southeast coast of India is endowed with a variety of biotopes such as neritic, estuarine, backwater and mangrove swamps (Figure1). At this consign an estuary named "Vellar" debouches into the Bay of Bengal, where it's origin is in the
Shervaroyan hills of Salem district, $240 \mathrm{Km}$. west of Parangipettai. This estuary is 600 metres wide at its junction with the sea. This is a true estuary and is subjected to long term seasonal variations. During the northeast monsoon (October to December) it drains more of fresh water.

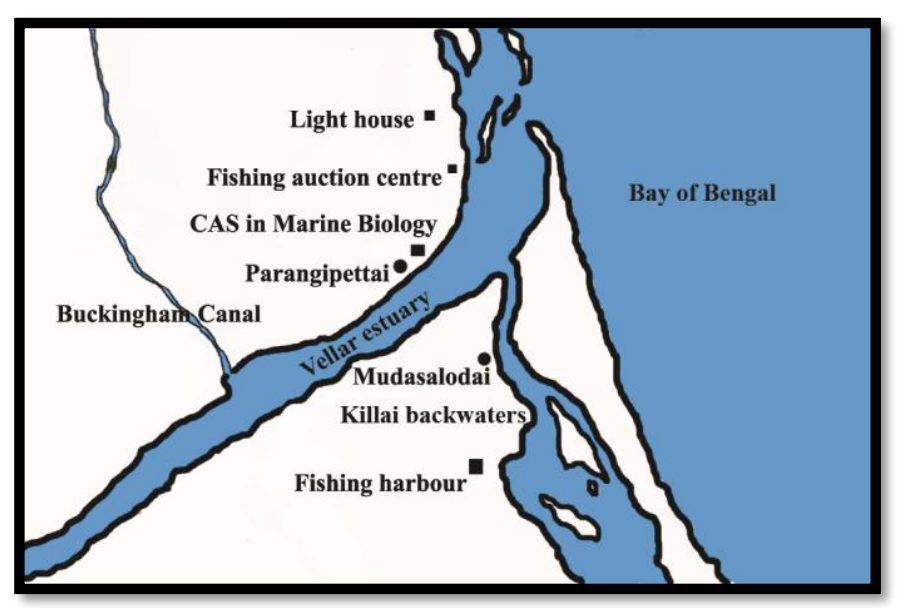

Figure 1: Map showing the study area

\subsection{Sample collection and processing}

Samples (fishes) were collected from the commercial fish landings at two popular landing centres namely Annankovil and Mudasalodai which are situated in the coastal waters of Parangipettai for a period of one year (January 2010-December 2010), no sampling was done in the month of May, due to the fishing holiday in the coast of Tamil Nadu. The collected fishes were kept in ice boxes and transferred to the laboratory and washed in tap water. The fishes were identified up to the species level. FAO species identification sheets for fishery purposes (EASTERN INDIAN OCEAN fishing area 57 and WESTERN CENTRAL PACIFIC fishing area 71) were used for species level identification. For further studies specimens were preserved in 5\% formalin after identification. Mechanized trawlers, mechanized boats, catamarans, plank-built boats and dugout canoes were mainly employed for fishing. Gill nets $(35 \mathrm{~mm})$ and trawl nets $(80 \mathrm{~cm}-$ $10 \mathrm{~cm}$ ) are commonly used gears in the study area. 


\subsection{Identification flowchart}

1. Wash the fish to be identified and remove all sand/sediment adhering to. Make sure that the fish is not damaged so as to obliterate the morphological configuration (do no prefer any spoiled or deteriorated fish).

2. To familiarise yourself with all measurements with the help of pictorial presentations.

3. Look for any conspicuous/predominant characters such as the presence of operculum, type of caudal fin, nature of dorsal fin and spine, lateral line, scutes, dark blotches, nature of snout, possessed by the fish. On successful examination, we could arrive tentatively at the family to which the given fish may be belonging.

4. After arriving at the family level, examine the list of species given under each family. Compare the character of the collected species with characters for each species under that family. While doing so, it is better to stick on to the meristic characters.

\section{RESULTS AND DISCUSSION}

A total of 447 individuals were collected and 93 species belonging to 57 genera and 42 families were identified (Table 1). About 97 species of teleost fishes were identified from Parangipettai waters [4]. In the present study, the catch and the fishery contributed varied between months. The family Carangidae ranked first with a maximum number of 7 species followed by Synodontidae ( 6 species), Engraulidae ( 6 species), Mugilidae (5 species), Leiognathidae (5 species) and Lutjanidae (4 species). All the species recorded were not observed in all the months. A total of 254 species belonging to 17 families were recorded in the Indian Ocean [5]. Of all the 93species recorded, the representatives of Clupeiformes (Nematalosa nasus), Gonorynchiformes (Chanos chanos), Siluriformes (Arius subrostratus, Plotosus canius), Synodontiformes (Saurida tumbil), Beloniformes (Hemiramphus far), Mugiliformes (all mugilids in table.1), Perciformes (Lates calcarifer. Epinephelus tauvina, E. bleekeri, Sillago sihama, Caranx para, Lutjanus argentimaculatus, Leiognathus splendens, Eluthronema tetradactylum, Kthala axillaris, Terapon puta, Etroplus suratensis, Scatophagus argus) and Pleuronectiformes (Pseudorhombus triocellatus) were present in the sampling site throughout the year. The fishery in this study area was not only supported from marine, but also from the adjacent estuarine waters, where the river Vellar joins the Parangipettai coastal waters. The presence of the above representatives throughout the year indicates their ability to withstand in any adverse conditions.

The number of species found in all the months fluctuated seasonally. This may be due to the increase in the fishing efforts as the fishermen are going to deeper regions for fishing, as a result the fleet may migrate to faraway places. On the other hand, the influence of biological processes also tends the animals to migrate to another fishing ground.

All the species observed in this study were of having commercial importance. They are fetching more cost by means of export at times of heap landings. Several studies were done on the biodiversity of marine fishes in the coasts of Tamil Nadu. Similar results were obtained in the study taken in Puducherry coastal waters by [6]. For the Eastern Indian ocean where the study area is situated, works on such aspects are scanty.
A total of 885 species belonging to 150 families were recorded in the South-Western Indian Ocean [7]. However, such kind of works are not much of interest in the Indian context, worldwide several works had been done. About 107 fish species belonging to 32 families have been reported from Malaysian waters [8]. 53 species from 13 families were also been observed in the streams of Sao Paulo state [9].

The number of species in this checklist is not final as there might be changes regarding the valid fish names in the future or new recorded species. Since Parangipettai coast is having close connections with the backwaters and small tributaries, there may be chances for some more species to enter in these waters, which may increase the numbers in this list. Optimistically this checklist will be a good reference for current and future studies.

\section{ACKNOWLEDGEMENTS}

We would like to thank to a mysterious referee for comments which helped to perk up the manuscript. Also, we thank the Director, CAS in Marine Biology, Annamalai University for the constant support and help in carrying out the study. One of the author would like to extend thanks to Research Management Centre, IIUM for their support through RIGS, IIUM. REFERENCES

[1] FAO. 2000. The state of World Fisheries and Aquaculture 2000. Food and Agriculture Organization of the United Nations, Rome, 142.

[2] Srinath, M., kuraikose, S.M.S., Ammini, P.L., Prasad, C. J., Ramani, K., Beena, M.K. 2006. Marine fish landings in India, 1985-2004. Estuar \& Tren. $161 \mathrm{p}$.

[3] Bensam, P. 2000. An introduction to demersal fin fish resources; In: V.N. Pillai and N.G. Menon (ed.). Marine fisheries research and management., CMFRI, Cochin. 495-498.

[4] Khan, S.A., Murugan, S. 2006. Manual on Identification of fishes, shrimps, lobsters and brachyuran crabs from Parangipettai waters. CAS in Marine Biology, Annamalai University, 148.

[5] Yogamoorthi, A. 2010. Marine Fish Diversity of Puducherry Coastal Waters. In: Balasubramanian, T. and S. Ajmal Khan (ed.). ENVIS, CAS in Marine Biology, Faculty of Marine Science, Annamalai University. ENVIS Publication Series., I. 222.

[6] Fricke, R. 1999. Fishes of the Mascarene Islands (Reunion, Mauritius, Rodrigues); An annotated Checklist, with Description of new species. Koenigstein: Koeltz scientific Books., 791.

[7] Letourneur, Y., Chabanet, P., Durville, P., Taquet, M., Teissier, E., Parmentier, M., Quero, J.C., Pothin, K. 2004. An Updated Checklist of the Marine fish fauna of the Reunion Island, South-west Indian Ocean. Cybium., 28 (3), 199-216.

[8] Hashim, Z.H., Zainuddin, R.Y., Shah, A.S.R.M., Sah, S.A.M., Mohammad, S., Mansor, M. 2012. Fish Checklist of Perak River, Malaysia. Check List, 8 (3), 408-413.

Cetra, M., Barella, W., Neto, F.L., Martins, A.G., Mello, B.J., Almeida, R.S. 2012. Fish fauna of the headwater streams that cross the Atlantic forest of South Sao Paulo state. Check List., 8 (3), 421-425.

Table 1: Month wise data on list of fishes observed from the study area during the year 2010-2011. (x: present; - : absent; N: No sampling due to fishing holiday)

\begin{tabular}{|c|c|c|c|c|c|c|c|c|c|c|c|c|}
\hline Species & Jan & Feb & Mar & Apr & May & Jun & Jul & Aug & Sep & Oct & Nov & Dec \\
\hline \multicolumn{13}{|l|}{ Elopiformes } \\
\hline \multicolumn{13}{|l|}{ Elopidae } \\
\hline Elops machnata (Forsskal, 1775) & $\mathrm{x}$ & $\mathrm{x}$ & $\mathrm{x}$ & $\mathrm{x}$ & $\mathrm{N}$ & $\mathrm{x}$ & $\mathrm{x}$ & $\mathrm{x}$ & $\mathrm{x}$ & $\mathrm{x}$ & $\mathrm{x}$ & - \\
\hline \multicolumn{13}{|l|}{ Megalopidae } \\
\hline Megalops cyprinoides (Broussonet, 1782) & - & $\mathrm{x}$ & $\mathrm{x}$ & $\mathrm{x}$ & $\mathrm{N}$ & $\mathrm{x}$ & $\mathrm{x}$ & $\mathrm{x}$ & - & $\mathrm{x}$ & - & $\mathrm{x}$ \\
\hline \multicolumn{13}{|l|}{ Anguilliformes } \\
\hline \multicolumn{13}{|l|}{ Anguillidae } \\
\hline Anguilla bicolor bicolor McClelland,1844 & - & - & - & - & $\mathrm{N}$ & - & - & - & - & $\mathrm{x}$ & $\mathrm{x}$ & $\mathrm{x}$ \\
\hline \multicolumn{13}{|l|}{ Muraenesocidae } \\
\hline Muraenesox bagio (Hamilton-Buchanan, 1822) & - & - & $\mathrm{x}$ & $\mathrm{x}$ & $\mathrm{N}$ & $\mathrm{x}$ & $\mathrm{x}$ & $\mathrm{x}$ & $\mathrm{x}$ & $\mathrm{x}$ & $\mathrm{x}$ & $\mathrm{x}$ \\
\hline Muraenesox cinereus (Forsskal, 1775) & $\mathrm{x}$ & $\mathrm{x}$ & $\mathrm{x}$ & $\mathrm{x}$ & $\mathrm{N}$ & $\mathrm{x}$ & $\mathrm{x}$ & $\mathrm{x}$ & $\mathrm{x}$ & - & - & - \\
\hline \multicolumn{13}{|l|}{ Clupeiformes } \\
\hline \multicolumn{13}{|l|}{ Chirocentridae } \\
\hline Chirocentrus dorab (Forsskal, 1775) & - & - & - & $\mathrm{x}$ & $\mathrm{N}$ & $\mathrm{x}$ & $\mathrm{x}$ & $\mathrm{x}$ & $\mathrm{x}$ & $\mathrm{x}$ & $\mathrm{x}$ & $\mathrm{x}$ \\
\hline
\end{tabular}




\begin{tabular}{|c|c|c|c|c|c|c|c|c|c|c|c|c|}
\hline \multicolumn{13}{|l|}{ Engraulidae } \\
\hline Thryssa malabarica (Bloch, 1795) & $\mathrm{x}$ & $\mathrm{x}$ & - & $\mathrm{x}$ & $\mathrm{N}$ & $\mathrm{x}$ & - & $\mathrm{x}$ & $\mathrm{x}$ & - & - & $\mathrm{x}$ \\
\hline Thryssa mystax (Schneider, 1801) & $\mathrm{x}$ & $\mathrm{x}$ & $\mathrm{x}$ & $\mathrm{x}$ & $\mathrm{N}$ & $\mathrm{x}$ & $\mathrm{x}$ & $\mathrm{x}$ & $\mathrm{x}$ & - & $\mathrm{x}$ & $\mathrm{x}$ \\
\hline Thryssa setirostris (Broussonet, 1782) & - & $\mathrm{x}$ & $\mathrm{x}$ & $\mathrm{x}$ & $\mathrm{N}$ & $\mathrm{x}$ & $\mathrm{x}$ & $\mathrm{x}$ & $\mathrm{x}$ & $\mathrm{x}$ & $\mathrm{x}$ & $\mathrm{x}$ \\
\hline Ciolia dussumieri (Valenciennes, 1848) & - & - & - & $\mathrm{x}$ & $\mathrm{N}$ & $\mathrm{x}$ & $\mathrm{x}$ & $\mathrm{x}$ & $\mathrm{x}$ & - & - & - \\
\hline Stolephorus commersonii Lacepede, 1803 & $\mathrm{x}$ & $\mathrm{x}$ & $\mathrm{x}$ & $\mathrm{x}$ & $\mathrm{N}$ & $\mathrm{x}$ & $\mathrm{x}$ & $\mathrm{x}$ & $\mathrm{x}$ & - & - & - \\
\hline Stolephorus waiteii Jordan \& Scale, 1926 & $\mathrm{x}$ & $\mathrm{x}$ & $\mathrm{x}$ & $\mathrm{x}$ & $\mathrm{N}$ & $\mathrm{x}$ & $\mathrm{x}$ & $\mathrm{x}$ & $\mathrm{x}$ & - & - & - \\
\hline \multicolumn{13}{|l|}{ Clupeidae } \\
\hline Sardinella albella (Valenciennes, 1847) & $\mathrm{x}$ & $\mathrm{x}$ & $\mathrm{x}$ & $\mathrm{x}$ & $\mathrm{N}$ & $\mathrm{x}$ & $\mathrm{x}$ & $\mathrm{x}$ & $\mathrm{x}$ & - & - & - \\
\hline Sardinella longiceps (Valenciennes, 1847) & $\mathrm{x}$ & $\mathrm{x}$ & $\mathrm{x}$ & $\mathrm{x}$ & $\mathrm{N}$ & $\mathrm{x}$ & $\mathrm{x}$ & $\mathrm{x}$ & - & - & - & - \\
\hline Nematalosa nasus (Bloch, 1795) & $\mathrm{x}$ & $\mathrm{x}$ & $\mathrm{x}$ & $\mathrm{x}$ & $\mathrm{N}$ & $\mathrm{x}$ & $\mathrm{x}$ & $\mathrm{x}$ & $\mathrm{x}$ & $\mathrm{x}$ & $\mathrm{x}$ & $\mathrm{x}$ \\
\hline \multicolumn{13}{|l|}{ Gonorynchiformes } \\
\hline \multicolumn{13}{|l|}{ Chanidae } \\
\hline Chanos chanos (Forsskal, 1775) & $\mathrm{x}$ & $\mathrm{x}$ & $\mathrm{x}$ & $\mathrm{x}$ & $\mathrm{N}$ & $\mathrm{x}$ & $\mathrm{x}$ & $\mathrm{x}$ & $\mathrm{x}$ & $\mathrm{x}$ & $\mathrm{x}$ & $\mathrm{x}$ \\
\hline \multicolumn{13}{|l|}{ Siluriformes } \\
\hline Aridae & & & & & & & & & & & & \\
\hline Arius maculates (Thunberg, 1792) & $\mathrm{x}$ & $\mathrm{x}$ & $\mathrm{x}$ & $\mathrm{x}$ & $\mathrm{N}$ & $\mathrm{x}$ & $\mathrm{x}$ & - & $\mathrm{x}$ & $\mathrm{x}$ & $\mathrm{x}$ & $\mathrm{x}$ \\
\hline Arius subrostratus (Valenciennes, 1840) & $\mathrm{x}$ & $\mathrm{x}$ & $\mathrm{x}$ & $\mathrm{x}$ & $\mathrm{N}$ & $\mathrm{x}$ & $\mathrm{x}$ & $\mathrm{x}$ & $\mathrm{x}$ & $\mathrm{x}$ & $\mathrm{x}$ & $\mathrm{x}$ \\
\hline Plotosidae & & & & & & & & & & & & \\
\hline Plotosus canius (Hamilton \& Buchanan, 1822) & $\mathrm{x}$ & $\mathrm{x}$ & $\mathrm{x}$ & $\mathrm{x}$ & $\mathrm{N}$ & $\mathrm{x}$ & $\mathrm{x}$ & $\mathrm{x}$ & $\mathrm{x}$ & $\mathrm{x}$ & $\mathrm{x}$ & $\mathrm{x}$ \\
\hline Aulopiformes & & & & & & & & & & & & \\
\hline Synodontidae & & & & & & & & & & & & \\
\hline Saurida tumbil (Bloch, 1745) & $\mathrm{x}$ & $\mathrm{x}$ & $\mathrm{x}$ & $\mathrm{x}$ & $\mathrm{N}$ & $\mathrm{x}$ & $\mathrm{x}$ & $\mathrm{x}$ & $\mathrm{x}$ & $\mathrm{x}$ & $\mathrm{x}$ & $\mathrm{x}$ \\
\hline Saurida undosquamis (Richardson, 1848) & $\mathrm{x}$ & $\mathrm{x}$ & - & - & $\mathrm{N}$ & $\mathrm{x}$ & $\mathrm{x}$ & $\mathrm{x}$ & $\mathrm{x}$ & $\mathrm{x}$ & $\mathrm{x}$ & $\mathrm{x}$ \\
\hline Saurida isarankurai (Shindo \& Yamada, 1972) & - & - & - & $\mathrm{x}$ & $\mathrm{N}$ & $\mathrm{x}$ & $\mathrm{x}$ & $\mathrm{x}$ & $\mathrm{x}$ & - & - & - \\
\hline Synodus indicus (Day, 1873) & - & - & - & - & $\mathrm{N}$ & - & - & $\mathrm{x}$ & $\mathrm{x}$ & $\mathrm{x}$ & $\mathrm{x}$ & - \\
\hline Synodus hoshinonis Tanaka, 1917 & $\mathrm{x}$ & $\mathrm{x}$ & - & - & $\mathrm{N}$ & - & - & - & $\mathrm{x}$ & $\mathrm{x}$ & $\mathrm{x}$ & $\mathrm{x}$ \\
\hline Trachinocephalus myops (Forster, 1801) & $\mathrm{x}$ & - & - & - & $\mathrm{N}$ & - & $\mathrm{x}$ & $\mathrm{x}$ & $\mathrm{x}$ & $\mathrm{x}$ & $\mathrm{x}$ & $\mathrm{x}$ \\
\hline Beloniformes & & & & & & & & & & & & \\
\hline Hemiramphidae & & & & & & & & & & & & \\
\hline Hemiramphus far (Forsskal, 1775) & $\mathrm{x}$ & $\mathrm{x}$ & $\mathrm{x}$ & - & $\mathrm{N}$ & $\mathrm{x}$ & $\mathrm{x}$ & $\mathrm{x}$ & $\mathrm{x}$ & $\mathrm{x}$ & $\mathrm{x}$ & $\mathrm{x}$ \\
\hline Hyporamphus limpatus (Valenciennes, 1846) & $\mathrm{x}$ & - & - & $\mathrm{x}$ & $\mathrm{N}$ & $\mathrm{x}$ & $\mathrm{x}$ & $\mathrm{x}$ & $\mathrm{x}$ & $\mathrm{x}$ & - & - \\
\hline Mugiliformes & & & & & & & & & & & & \\
\hline Mugilidae & & & & & & & & & & & & \\
\hline Mugil cephalus (Linnaeus, 1758) & $\mathrm{x}$ & $\mathrm{x}$ & $\mathrm{x}$ & $\mathrm{x}$ & $\mathrm{N}$ & $\mathrm{x}$ & $\mathrm{x}$ & $\mathrm{x}$ & $\mathrm{x}$ & $\mathrm{x}$ & $\mathrm{x}$ & $\mathrm{x}$ \\
\hline Liza parsia (Hamilton \& Buchanan, 1822) & $\mathrm{x}$ & $\mathrm{x}$ & $\mathrm{x}$ & $\mathrm{x}$ & $\mathrm{N}$ & $\mathrm{x}$ & $\mathrm{x}$ & $\mathrm{x}$ & $\mathrm{x}$ & $\mathrm{x}$ & $\mathrm{x}$ & $\mathrm{x}$ \\
\hline Liza tade (Forsskal, 1775) & $\mathrm{x}$ & $\mathrm{x}$ & $\mathrm{x}$ & $\mathrm{x}$ & $\mathrm{N}$ & $\mathrm{x}$ & $\mathrm{x}$ & $\mathrm{x}$ & $\mathrm{x}$ & $\mathrm{x}$ & $\mathrm{x}$ & $\mathrm{x}$ \\
\hline Liza subviridis (Valenciennes, 1876) & $\mathrm{x}$ & $\mathrm{x}$ & $\mathrm{x}$ & $\mathrm{x}$ & $\mathrm{N}$ & $\mathrm{x}$ & $\mathrm{x}$ & $\mathrm{x}$ & $\mathrm{x}$ & $\mathrm{x}$ & $\mathrm{x}$ & $\mathrm{x}$ \\
\hline Valamugil cunnesius (Valenciennes, 1836) & $\mathrm{x}$ & $\mathrm{x}$ & $\mathrm{x}$ & $\mathrm{x}$ & $\mathrm{N}$ & $\mathrm{x}$ & $\mathrm{x}$ & $\mathrm{x}$ & $\mathrm{x}$ & $\mathrm{x}$ & $\mathrm{x}$ & $\mathrm{x}$ \\
\hline Perciformes & & & & & & & & & & & & \\
\hline Ambassidae & & & & & & & & & & & & \\
\hline Ambassis gymnocephalus (Lacepede, 1801) & $\mathrm{x}$ & $\mathrm{x}$ & $\mathrm{x}$ & $\mathrm{x}$ & $\mathrm{N}$ & $\mathrm{x}$ & $\mathrm{x}$ & $\mathrm{x}$ & $\mathrm{x}$ & - & - & - \\
\hline Centropomidae & & & & & & & & & & & & \\
\hline Lates calcarifer (Bloch, 1790$)$ & $\mathrm{x}$ & $\mathrm{x}$ & $\mathrm{x}$ & $\mathrm{x}$ & $\mathrm{N}$ & $\mathrm{x}$ & $\mathrm{x}$ & $\mathrm{x}$ & $\mathrm{x}$ & $\mathrm{x}$ & $\mathrm{x}$ & $\mathrm{x}$ \\
\hline Serranidae & & & & & & & & & & & & \\
\hline Epinepehlus tauvina (Forsskal, 1775) & $\mathrm{x}$ & $\mathrm{x}$ & $\mathrm{x}$ & $\mathrm{x}$ & $\mathrm{N}$ & $\mathrm{x}$ & $\mathrm{x}$ & $\mathrm{x}$ & $\mathrm{x}$ & $\mathrm{x}$ & $\mathrm{x}$ & $\mathrm{x}$ \\
\hline Epinephelus bleekeri (Vaillant, 1877) & $\mathrm{x}$ & $\mathrm{x}$ & $\mathrm{x}$ & $\mathrm{x}$ & $\mathrm{N}$ & $\mathrm{x}$ & $\mathrm{x}$ & $\mathrm{x}$ & $\mathrm{x}$ & $\mathrm{x}$ & $\mathrm{x}$ & $\mathrm{x}$ \\
\hline Epinephelus malabaricus (Schneider, 1801) & $\mathrm{x}$ & $\mathrm{x}$ & $\mathrm{x}$ & - & $\mathrm{N}$ & - & - & - & $\mathrm{x}$ & $\mathrm{x}$ & $\mathrm{x}$ & $\mathrm{x}$ \\
\hline Lactaridae & & & & & & & & & & & & \\
\hline Lactarius lactarius (Bloch \& Schneider, 1801) & $\mathrm{x}$ & $\mathrm{x}$ & - & - & $\mathrm{N}$ & - & - & - & $\mathrm{x}$ & $\mathrm{x}$ & $\mathrm{x}$ & $\mathrm{x}$ \\
\hline Sillaginidae & & & & & & & & & & & & \\
\hline Sillago sihama (Forsskal, 1775) & $\mathrm{x}$ & $\mathrm{x}$ & $\mathrm{x}$ & $\mathrm{x}$ & $\mathrm{N}$ & $\mathrm{x}$ & $\mathrm{x}$ & $\mathrm{x}$ & $\mathrm{x}$ & $\mathrm{x}$ & $\mathrm{x}$ & $\mathrm{x}$ \\
\hline Carangidae & & & & & & & & & & & & \\
\hline Alectis indicus (Ruppel, 1830) & $\mathrm{x}$ & $\mathrm{x}$ & $\mathrm{x}$ & $\mathrm{x}$ & $\mathrm{N}$ & $\mathrm{x}$ & $\mathrm{x}$ & $\mathrm{x}$ & $\mathrm{x}$ & - & - & - \\
\hline Carangoides malabaricus (Bloch \& Schneider, 1801) & $\mathrm{x}$ & $\mathrm{x}$ & $\mathrm{x}$ & $\mathrm{x}$ & $\mathrm{N}$ & $\mathrm{x}$ & $\mathrm{x}$ & $\mathrm{x}$ & $\mathrm{x}$ & $\mathrm{x}$ & $\mathrm{x}$ & - \\
\hline Caranx ignobilis (Forsskal, 1775) & $\mathrm{x}$ & $\mathrm{x}$ & $\mathrm{x}$ & $\mathrm{x}$ & $\mathrm{N}$ & $\mathrm{x}$ & $\mathrm{x}$ & $\mathrm{x}$ & $\mathrm{x}$ & - & $\mathrm{x}$ & $\mathrm{x}$ \\
\hline Caranx para Cuvier, 1833 & $\mathrm{x}$ & $\mathrm{x}$ & $\mathrm{x}$ & $\mathrm{x}$ & $\mathrm{N}$ & $\mathrm{x}$ & $\mathrm{x}$ & $\mathrm{x}$ & $\mathrm{x}$ & $\mathrm{x}$ & $\mathrm{x}$ & $\mathrm{x}$ \\
\hline Caranx sexfasciatus (Quoy \& Gaimard, 1824) & $\mathrm{x}$ & $\mathrm{x}$ & $\mathrm{x}$ & $\mathrm{x}$ & $\mathrm{N}$ & $\mathrm{x}$ & $\mathrm{x}$ & $\mathrm{x}$ & $\mathrm{x}$ & - & $\mathrm{x}$ & $\mathrm{x}$ \\
\hline Parastromateus niger (Bloch, 1795) & $\mathrm{x}$ & - & - & & $\mathrm{N}$ & - & $\mathrm{x}$ & $\mathrm{x}$ & $\mathrm{x}$ & - & - & - \\
\hline Scomberoides tol (Cuvier, 1832) & $\mathrm{x}$ & $\mathrm{x}$ & $\mathrm{x}$ & $\mathrm{x}$ & $\mathrm{N}$ & $\mathrm{x}$ & $\mathrm{x}$ & $\mathrm{x}$ & $\mathrm{x}$ & - & - & - \\
\hline Lutjanidae & & & & & & & & & & & & \\
\hline Lutjanus argentimaculatus (Forsskal, 1975) & $\mathrm{x}$ & $\mathrm{x}$ & $\mathrm{x}$ & $\mathrm{x}$ & $\mathrm{N}$ & $\mathrm{x}$ & $\mathrm{x}$ & $\mathrm{x}$ & $\mathrm{x}$ & $\mathrm{x}$ & $\mathrm{x}$ & $\mathrm{x}$ \\
\hline Lutjanus fulviflammus (Forsskal, 1775) & $\mathrm{x}$ & $\mathrm{x}$ & $\mathrm{x}$ & $\mathrm{x}$ & $\mathrm{N}$ & $\mathrm{x}$ & $\mathrm{x}$ & $\mathrm{x}$ & $\mathrm{x}$ & - & - & $\mathrm{x}$ \\
\hline Lutjanus johni (Bloch, 1792) & $\mathrm{x}$ & $\mathrm{x}$ & $\mathrm{x}$ & $\mathrm{x}$ & $\mathrm{N}$ & $\mathrm{x}$ & $\mathrm{x}$ & $\mathrm{x}$ & $\mathrm{x}$ & $\mathrm{x}$ & - & - \\
\hline Lutjanus russelli (Bleeker, 1849) & - & - & $\mathrm{x}$ & $\mathrm{x}$ & $\mathrm{N}$ & $\mathrm{x}$ & $\mathrm{x}$ & $\mathrm{x}$ & $\mathrm{x}$ & $\mathrm{x}$ & $\mathrm{x}$ & $\mathrm{x}$ \\
\hline Leiognathidae & & & & & & & & & & & & \\
\hline Leiognathus splendens (Cuvier, 1821) & $\mathrm{x}$ & $\mathrm{x}$ & $\mathrm{x}$ & $\mathrm{x}$ & $\mathrm{N}$ & $\mathrm{x}$ & $\mathrm{x}$ & $\mathrm{x}$ & $\mathrm{x}$ & $\mathrm{x}$ & $\mathrm{x}$ & $\mathrm{x}$ \\
\hline Leiognathus equulus (Forsskal, 1775) & $\mathrm{x}$ & $\mathrm{x}$ & $\mathrm{x}$ & $\mathrm{x}$ & $\mathrm{N}$ & $\mathrm{x}$ & $\mathrm{x}$ & $\mathrm{x}$ & $\mathrm{x}$ & $\mathrm{x}$ & - & - \\
\hline Leiognathus bindus (Valenciennes, 1835) & - & - & $\mathrm{x}$ & $\mathrm{x}$ & $\mathrm{N}$ & $\mathrm{x}$ & $\mathrm{x}$ & $\mathrm{x}$ & $\mathrm{x}$ & $\mathrm{x}$ & $\mathrm{x}$ & $\mathrm{x}$ \\
\hline Secutor insidiator (Bloch, 1787) & - & $\mathrm{x}$ & $\mathrm{x}$ & $\mathrm{x}$ & $\mathrm{N}$ & $\mathrm{x}$ & $\mathrm{x}$ & $\mathrm{x}$ & $\mathrm{x}$ & $\mathrm{x}$ & - & - \\
\hline Secutor ruconius (Hamilton-Buchanan, 1822) & - & - & $\mathrm{x}$ & $\mathrm{x}$ & $\mathrm{N}$ & $\mathrm{x}$ & $\mathrm{x}$ & $\mathrm{x}$ & $\mathrm{x}$ & $\mathrm{x}$ & $\mathrm{x}$ & $\mathrm{x}$ \\
\hline Polynemidae & & & & & & & & & & & & \\
\hline Eluthronema tetradactylum (Shaw, 1804) & $\mathrm{x}$ & $\mathrm{x}$ & $\mathrm{x}$ & $\mathrm{x}$ & $\mathrm{N}$ & $\mathrm{x}$ & $\mathrm{x}$ & $\mathrm{x}$ & $\mathrm{x}$ & $\mathrm{x}$ & $\mathrm{x}$ & $\mathrm{x}$ \\
\hline Polynemus plebius Broussonet, 1782 & $\mathrm{x}$ & $\mathrm{x}$ & $\mathrm{x}$ & $\mathrm{x}$ & $\mathrm{N}$ & $\mathrm{x}$ & $\mathrm{x}$ & - & - & - & - & - \\
\hline Haemulidae & & & & & & & & & & & & \\
\hline Pomadasys kakkan (Cuvier, 1830) & - & - & - & $\mathrm{x}$ & $\mathrm{N}$ & $\mathrm{x}$ & $\mathrm{x}$ & $\mathrm{x}$ & $\mathrm{x}$ & $\mathrm{x}$ & $\mathrm{x}$ & - \\
\hline
\end{tabular}




\begin{tabular}{|c|c|c|c|c|c|c|c|c|c|c|c|c|}
\hline Pomadasys maculatum (Bloch, 1979) & $\mathrm{x}$ & $\mathrm{x}$ & $\mathrm{x}$ & $\mathrm{x}$ & $\mathrm{N}$ & $\mathrm{x}$ & $\mathrm{x}$ & $\mathrm{x}$ & $\mathrm{x}$ & - & - & - \\
\hline \multicolumn{13}{|l|}{ Sciaenidae } \\
\hline Kthala axillaris (Cuvier, 1830) & $\mathrm{x}$ & $\mathrm{x}$ & $\mathrm{x}$ & $\mathrm{x}$ & $\mathrm{N}$ & $\mathrm{x}$ & $\mathrm{x}$ & $\mathrm{x}$ & $\mathrm{x}$ & $\mathrm{x}$ & $\mathrm{x}$ & $\mathrm{x}$ \\
\hline Johnius corutta Bloch, 1793 & $\mathrm{x}$ & $\mathrm{x}$ & $\mathrm{x}$ & $\mathrm{x}$ & $\mathrm{N}$ & $\mathrm{x}$ & $\mathrm{x}$ & $\mathrm{x}$ & $\mathrm{x}$ & $\mathrm{x}$ & - & - \\
\hline Dendrophysa russelli (Cuvier, 1830) & - & - & - & $\mathrm{x}$ & $\mathrm{N}$ & $\mathrm{x}$ & $\mathrm{x}$ & $\mathrm{x}$ & $\mathrm{x}$ & - & - & - \\
\hline \multicolumn{13}{|l|}{ Lethrinidae } \\
\hline Lethrinus nebulosus (Forsskal, 1775) & $\mathrm{x}$ & $\mathrm{x}$ & $\mathrm{x}$ & $\mathrm{x}$ & $\mathrm{N}$ & $\mathrm{x}$ & $\mathrm{x}$ & $\mathrm{x}$ & $\mathrm{x}$ & - & - & - \\
\hline \multicolumn{13}{|l|}{ Nemipteridae } \\
\hline Nemipterus bleekeri (Day, 1875) & - & - & - & - & $\mathrm{N}$ & $\mathrm{x}$ & $\mathrm{x}$ & $\mathrm{x}$ & $\mathrm{x}$ & $\mathrm{x}$ & $\mathrm{x}$ & $\mathrm{x}$ \\
\hline Nemipterus japonicus (Bloch, 1791) & - & - & - & - & $\mathrm{N}$ & $\mathrm{x}$ & $\mathrm{x}$ & $\mathrm{x}$ & $\mathrm{x}$ & $\mathrm{x}$ & $\mathrm{x}$ & $\mathrm{x}$ \\
\hline \multicolumn{13}{|l|}{ Gerreidae } \\
\hline Gerres abbreviatus (Bleeker, 1850) & - & $\mathrm{x}$ & $\mathrm{x}$ & $\mathrm{x}$ & $\mathrm{N}$ & $\mathrm{x}$ & $\mathrm{x}$ & $\mathrm{x}$ & $\mathrm{x}$ & - & - & - \\
\hline Gerres filamentosus (Cuvier, 1829) & $\mathrm{x}$ & $\mathrm{x}$ & $\mathrm{x}$ & $\mathrm{x}$ & $\mathrm{N}$ & $\mathrm{x}$ & $\mathrm{x}$ & $\mathrm{x}$ & $\mathrm{x}$ & - & - & - \\
\hline Gerres rappi (Barnard, 1927) & $\mathrm{x}$ & $\mathrm{x}$ & $\mathrm{x}$ & $\mathrm{x}$ & $\mathrm{N}$ & $\mathrm{x}$ & $\mathrm{x}$ & $\mathrm{x}$ & $\mathrm{x}$ & - & - & $\mathrm{x}$ \\
\hline \multicolumn{13}{|l|}{ Mullidae } \\
\hline Upeneus sulphureus (Cuvier, 1829) & $\mathrm{x}$ & $\mathrm{x}$ & $\mathrm{x}$ & $\mathrm{x}$ & $\mathrm{N}$ & - & - & - & - & $\mathrm{x}$ & $\mathrm{x}$ & $\mathrm{x}$ \\
\hline Upeneus vittatus (Forsskal, 1775) & - & - & - & $\mathrm{x}$ & $\mathrm{N}$ & $\mathrm{x}$ & $\mathrm{x}$ & $\mathrm{x}$ & $\mathrm{x}$ & $\mathrm{x}$ & $\mathrm{x}$ & $\mathrm{x}$ \\
\hline \multicolumn{13}{|l|}{ Stromatidae } \\
\hline Pampus argenteus (Euphrasen, 1788) & $\mathrm{x}$ & $\mathrm{x}$ & $\mathrm{x}$ & $\mathrm{x}$ & $\mathrm{N}$ & $\mathrm{x}$ & $\mathrm{x}$ & $\mathrm{x}$ & $\mathrm{x}$ & - & - & - \\
\hline Pampus chinensis (Euphrasen, 1788) & - & - & - & $\mathrm{x}$ & $\mathrm{N}$ & $\mathrm{x}$ & $\mathrm{x}$ & $\mathrm{x}$ & $\mathrm{x}$ & $\mathrm{x}$ & - & - \\
\hline \multicolumn{13}{|l|}{ Scombridae } \\
\hline Rastrelliger kanagurta (Cuvier, 1817) & - & - & $\mathrm{x}$ & $\mathrm{x}$ & $\mathrm{N}$ & $\mathrm{x}$ & $\mathrm{x}$ & $\mathrm{x}$ & $\mathrm{x}$ & $\mathrm{x}$ & - & - \\
\hline Scomberomorus guttatus (Bloch \& Schneider, 1801) & $\mathrm{x}$ & $\mathrm{x}$ & $\mathrm{x}$ & - & $\mathrm{N}$ & - & - & - & - & $\mathrm{x}$ & $\mathrm{x}$ & $\mathrm{x}$ \\
\hline Scomberomorus commerson (Lacepede, 1801) & - & - & - & $\mathrm{x}$ & $\mathrm{N}$ & $\mathrm{x}$ & $\mathrm{x}$ & $\mathrm{x}$ & $\mathrm{x}$ & - & - & - \\
\hline Ethynnus affinis (Cantor, 1849) & - & $\mathrm{x}$ & $\mathrm{x}$ & $\mathrm{x}$ & $\mathrm{N}$ & $\mathrm{x}$ & $\mathrm{x}$ & $\mathrm{x}$ & $\mathrm{x}$ & - & - & - \\
\hline \multicolumn{13}{|l|}{ Sphyraenidae } \\
\hline Sphyraena barracuda (Walbaum, 1792) & $\mathrm{x}$ & $\mathrm{x}$ & $\mathrm{x}$ & $\mathrm{x}$ & $\mathrm{N}$ & - & - & - & - & - & $\mathrm{x}$ & $\mathrm{x}$ \\
\hline Sphyraena obtusata (Cuvier, 1829) & - & - & - & $\mathrm{x}$ & $\mathrm{N}$ & $\mathrm{x}$ & $\mathrm{x}$ & $\mathrm{x}$ & $\mathrm{x}$ & $\mathrm{x}$ & - & - \\
\hline \multicolumn{13}{|l|}{ Siganidae } \\
\hline Siganus javus (Linnaeus, 1766) & $\mathrm{x}$ & $\mathrm{x}$ & $\mathrm{x}$ & $\mathrm{x}$ & $\mathrm{N}$ & $\mathrm{x}$ & $\mathrm{x}$ & $\mathrm{x}$ & - & - & - & - \\
\hline Siganus canaliculatus (Park, 1797) & $\mathrm{x}$ & $\mathrm{x}$ & $\mathrm{x}$ & $\mathrm{x}$ & $\mathrm{N}$ & $\mathrm{x}$ & - & - & - & $\mathrm{x}$ & $\mathrm{x}$ & $\mathrm{x}$ \\
\hline \multicolumn{13}{|l|}{ Teraponidae } \\
\hline Terapon jarbua (Forsskal, 1775) & $\mathrm{x}$ & $\mathrm{x}$ & $\mathrm{x}$ & $\mathrm{x}$ & $\mathrm{N}$ & $\mathrm{x}$ & $\mathrm{x}$ & $\mathrm{x}$ & $\mathrm{x}$ & - & - & $\mathrm{x}$ \\
\hline Terapon puta (Cuvier, 1829) & $\mathrm{x}$ & $\mathrm{x}$ & $\mathrm{x}$ & $\mathrm{x}$ & $\mathrm{N}$ & $\mathrm{x}$ & $\mathrm{x}$ & $\mathrm{x}$ & $\mathrm{x}$ & $\mathrm{x}$ & $\mathrm{x}$ & $\mathrm{x}$ \\
\hline \multicolumn{13}{|l|}{ Trichiuridae } \\
\hline Trichiurus lepturus Linnaeus, 1758 & $\mathrm{x}$ & $\mathrm{x}$ & $\mathrm{x}$ & $\mathrm{x}$ & $\mathrm{N}$ & $\mathrm{x}$ & $\mathrm{x}$ & $\mathrm{x}$ & $\mathrm{x}$ & - & - & - \\
\hline \multicolumn{13}{|l|}{ Cichilidae } \\
\hline Etroplus suratensis (Bloch, 1797) & $\mathrm{x}$ & $\mathrm{x}$ & $\mathrm{x}$ & $\mathrm{x}$ & $\mathrm{N}$ & $\mathrm{x}$ & $\mathrm{x}$ & $\mathrm{x}$ & $\mathrm{x}$ & $\mathrm{x}$ & $\mathrm{x}$ & $\mathrm{x}$ \\
\hline \multicolumn{13}{|l|}{ Drepanidae } \\
\hline Drepane punctata (Linnaeus, 1758) & $\mathrm{x}$ & $\mathrm{x}$ & $\mathrm{x}$ & $\mathrm{x}$ & $\mathrm{N}$ & $\mathrm{x}$ & $\mathrm{x}$ & $\mathrm{x}$ & $\mathrm{x}$ & - & - & - \\
\hline \multicolumn{13}{|l|}{ Lobotidae } \\
\hline Lobotes surinamensis (Bloch, 1790) & $\mathrm{x}$ & $\mathrm{x}$ & $\mathrm{x}$ & $\mathrm{x}$ & $\mathrm{N}$ & $\mathrm{x}$ & $\mathrm{x}$ & $\mathrm{x}$ & $\mathrm{x}$ & - & - & - \\
\hline \multicolumn{13}{|l|}{ Acanthuridae } \\
\hline Acanthurus bleekeri Gunther, 1861 & $\mathrm{x}$ & $\mathrm{x}$ & $\mathrm{x}$ & $\mathrm{x}$ & $\mathrm{N}$ & $\mathrm{x}$ & $\mathrm{x}$ & $\mathrm{x}$ & $\mathrm{x}$ & - & - & - \\
\hline Scatophagidae & & & & & & & & & & & & \\
\hline Scatophagus argus (Bloch, 1788) & $\mathrm{x}$ & $\mathrm{x}$ & $\mathrm{x}$ & $\mathrm{x}$ & $\mathrm{N}$ & $\mathrm{x}$ & $\mathrm{x}$ & $\mathrm{x}$ & $\mathrm{x}$ & $\mathrm{x}$ & $\mathrm{x}$ & $\mathrm{x}$ \\
\hline Pleuronectiformes & & & & & & & & & & & & \\
\hline Bothidae & & & & & & & & & & & & \\
\hline Pseudorhombus triocellatus (Schneider, 1801) & $\mathrm{x}$ & $\mathrm{x}$ & $\mathrm{x}$ & $\mathrm{x}$ & $\mathrm{N}$ & $\mathrm{x}$ & $\mathrm{x}$ & $\mathrm{x}$ & $\mathrm{x}$ & $\mathrm{x}$ & $\mathrm{x}$ & $\mathrm{x}$ \\
\hline Pseudorhombus elevatus Ogilby, 1912 & $\mathrm{x}$ & $\mathrm{x}$ & $\mathrm{x}$ & $\mathrm{x}$ & $\mathrm{N}$ & $\mathrm{x}$ & $\mathrm{x}$ & $\mathrm{x}$ & - & - & - & - \\
\hline Cynoglossidae & & & & & & & & & & & & \\
\hline Cynoglossus puncticeps (Richardson, 1846) & $\mathrm{x}$ & $\mathrm{x}$ & - & $\mathrm{x}$ & $\mathrm{N}$ & $\mathrm{x}$ & $\mathrm{x}$ & $\mathrm{x}$ & $\mathrm{x}$ & $\mathrm{x}$ & - & - \\
\hline Cynoglossus arel (Schneider, 1801) & $\mathrm{x}$ & $\mathrm{x}$ & $\mathrm{x}$ & $\mathrm{x}$ & $\mathrm{N}$ & $\mathrm{x}$ & $\mathrm{x}$ & $\mathrm{x}$ & $\mathrm{x}$ & - & - & - \\
\hline Psettodidae & & & & & & & & & & & & \\
\hline Psettodes erumei (Schneider, 1801) & - & $\mathrm{x}$ & $\mathrm{x}$ & $\mathrm{x}$ & $\mathrm{N}$ & $\mathrm{x}$ & $\mathrm{x}$ & $\mathrm{x}$ & $\mathrm{x}$ & $\mathrm{x}$ & - & - \\
\hline
\end{tabular}

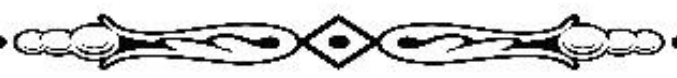

